\title{
LA PLANIFICACIÓN TURÍSTICA SUSTENTABLE DESDE UN ENFOQUE SISTÉMICO
}

\author{
Georgina del Socorro Medina Argueta \\ Universidad de Quintana Roo \\ México \\ geormedar@hotmail.com \\ Ángel Aarón Rosado Varela \\ Universidad Politécnica de Bacalar \\ México \\ Aaron.rosado@upb.edu.mx
}

\section{RESUMEN}

Se presenta un análisis de carácter teórico-conceptual sobre el Desarrollo Sustentable y la Planificación Turística desde un enfoque sistémico. El estudio se aborda bajo la Teoría General de Sistemas, específicamente en el contexto de los sistemas sociales complejos con enfoque sustentable. Este enfoque, permitió vislumbrar al turismo como un sistema complejo integrado por componentes estrechamente relacionados con el medio ambiente y la sociedad, que deben ser considerados para entender la importancia de la autoorganización, multidisciplinaridad, dinámica y evolución, determinantes de la sinergia entre los componentes de un sistema turístico sustentable.

Entre los resultados más destacados se encuentra que los componentes del sistema turístico sí pueden integrarse a partir de un enfoque sistémico permitiendo desarrollar la actividad turística bajo un enfoque sustentable debido a sus características, como la complejidad, dinámica, interdisciplinariedad, entre otros, lo que permite la adaptación de los principios de sustentabilidad. Es decir, comparten propiedades comunes y que a pesar de ser disciplinas diferentes, el enfoque sistémico permite explicar la dinámica del turismo aun con la dificultad en la comprensión de los elementos cuando se le atribuyen principios sustentables.

Palabras clave: Complejidad, sistemas, sustentabilidad, planificación, turismo. 


\title{
SUSTAINABLE TOURISM PLANNING FROM A SYSTEMIC APPROACH
}

\author{
Georgina del Socorro Medina Argueta \\ Universidad de Quintana Roo \\ México \\ geormedar@hotmail.com \\ Ángel Aarón Rosado Varela \\ Universidad Politécnica de Bacalar \\ México \\ Aaron.rosado@upb.edu.mx
}

\begin{abstract}
A theoretical and conceptual analysis of Sustainable Development and Tourism Planning from a systemic approach is presented. The research is conducted based on the General Systems Theory, specifically in the context of complex social systems with a sustainable approach. This approach allowed for the examination of tourism as a complex system composed of components closely related to the environment and society. These components need to be considered in order to understand the importance of selforganization, multidisciplinary, dynamic and evolution, as well as to determine synergy between components a sustainable tourism. The results confirm that the components of the tourism system can be integrated from a systemic approach, allowing conduct tourism development based on a sustainable approach due to features such as complexity, dynamic and interdisciplinary, amongst others that embrace principles of sustainability. As a conclusion this article argues that in spite of being different disciplines, the systemic approach allows for understanding the tourism sector dynamics.
\end{abstract}

Keywords: Complexity, systems, sustainability, planning, tourism. 


\section{INTRODUCCIÓN}

La planificación turística sustentable ha adquirido gran relevancia entre los actores que persiguen la actividad del turismo como una oportunidad para lograr un desarrollo económico además garantice un bienestar social y ambiental de los destinos. En este sentido, el enfoque sistémico permite que el turismo sea apreciado no solo como una actividad económica sino como un fenómeno social complejo y que por su naturaleza está caracterizado por elementos en común con los principios sustentables lo que significa una mejor comprensión en el momento de planificar y tomar decisiones determinantes como actores turísticos.

El trabajo tiene carácter analítico en su contenido y su objetivo es identificar los aspectos relevantes y las aproximaciones teóricas. Por lo que se realiza una revisión descriptiva y evaluativa bibliográfica de documentos primarios que en su contenido integren información directa acerca de la Teoría General de Sistemas y del Desarrollo Sustentable que permitan identificar las variables en común dentro de un sistema social complejo como lo es la actividad turística.

En los primeros apartados, se presenta como antecedente del pensamiento sistémico a la Teoría General de Sistemas y a los autores más destacados que han servido como aportadores a esta corriente. Asimismo describe la caracterización de los sistemas y los sistemas complejos en las organizaciones sociales destacando la importancia de la complejidad como característica común entre los sistemas sociales así como la forma de entender un sistema social en sus diferentes niveles dentro de la teoría de Niklas Luhmann. Seguidamente, la funcionalidad, efectividad y sinergia en los sistemas complejos se presentan como elementos ideales que debe integrar un sistema complejo, y a partir de ello, se establece la importancia de sus efectos positivos que puede causar la presencia en los sistemas.

Posterior, se diferencia a los términos de planeamiento y de planeamiento sistémico representándolos como ejemplos los modelos de planeación sistémica de Gharajedaghi y el de Schwaninger (en Herrscher, 2008: 238-240). En este sentido, se presenta la relación entre la visión sistémica y el desarrollo sustentable y se relaciona al turismo como ejemplo de sistema social complejo que puede estudiarse como un fenómeno con características sustentables. Asimismo, se conceptualiza el término "modelo" y explica la utilización y limitantes dentro de los sistemas complejos y como estos han permitido la explicación de la dinámica turística. 
A manera de integración de los temas de planificación turística sustentable desde un enfoque sistémico previos presentados, se describen brevemente los modelos de Leiper (1979), Cooper (1997), Jafari y McInchosh (1990), entre otros, como ejemplos de autores clásicos que han integrado modelos de sistemas para explicar la dinámica del turismo y se explica la relación entre el desarrollo sustentable y su importancia dentro de la planificación turística. Se define el concepto de desarrollo sustentable en el turismo y los cambios globales que originan la exigencia de los turistas en consumir productos turísticos que se resulten de una planificación ambiental y social.

Por ello, se identifican los niveles de sostenibilidad que debe integrar un desarrollo de actividades socioeconómicas como el turismo, se presenta la importancia de los indicadores sustentables dentro de la planificación del turismo sustentable así como las diversas organizaciones que han integrado indicadores de sostenibilidad para abordar los estudios del turismo a partir de los sistemas.

Por último, se presenta una conclusión general en la que se recuperan los conceptos y aspectos relevantes de la investigación a manera de análisis acerca del comportamiento sistémico, la complejidad dentro de los sistemas y de los sistemas dentro de la planificación turística con enfoque sustentable. 


\section{ANTECEDENTES DEL PENSAMIENTO SISTÉMICO.}

Dentro del pensamiento sistémico encontramos a la Teoría General de Sistemas como corriente principal, desarrollada por Bertalanffy a mediados del siglo XX. Entre los seguidores de esta teoría y quienes han realizado aportaciones en su desarrollo dentro del mismo periodo, se encuentran Foerster (1949), Gerard (1956), Maturana (1972 en Maturana 1997), Luhmann (1995), Boulding (1999), Parsons (1999), Rapoport (1985), Günther (2005), entre otros.

Esta teoría surgió debido a la necesidad de abordar científicamente la comprensión de los sistemas concretos que forman la realidad, generalmente complejos y únicos, resultantes de una historia particular. Para reforzar esta idea, Van Gigch (2001: 16) explica:

..."Los problemas que nos ocupan hoy en día bajo el rubro "sistema" no "nacieron ayer", son más bien expresiones contemporáneas de interrogantes perennes planteados y discutidos durante siglos". Asimismo, enfatiza que "Los problemas de sistemas, requieren soluciones de sistemas; lo que significa que debemos dirigirnos a resolver los problemas del sistema mayor, con conclusiones que satisfagan no sólo los objetivos de los subsistemas, sino también la sobrevivencia del sistema global"...

La Teoría General de Sistemas, es un esfuerzo de estudio interdisciplinario que trata de encontrar las propiedades comunes a entidades, los sistemas que se presentan en todos los niveles de la realidad, pero que son objetivo tradicionalmente de disciplinas académicas diferentes.

Sin embargo, es importante tener en cuenta que el pensamiento sistémico no constituye un conjunto orgánico de principios estandarizados que se imponga como un cuerpo doctrinado válido para toda la comunidad sistémica. Por el contrario, se privilegia la variedad, se acepta la ambigüedad y la contradicción, y se promueve el pensamiento crítico (Herrscher, 2008: 165).

Por lo antes mencionado, los principios y metodologías que intentan servir como recetas para el éxito total de un sistema, son en realidad intentos de diversos autores, en el que lejos de minimizar su trabajo y esfuerzos, son más bien alternativas que presentan a través de sus diversas disciplinas y escenarios en turno. Por ello, no se trata de seguir una u otra metodología, sino de encontrar la mejor alternativa que ofrezca aquellas herramientas pertinentes para nuestro objeto de estudio. A pesar de lo anterior, los sistemas comparten en la mayoría de los casos algunas características como la autorreferencia, interdependencia, interacción, recursividad y dinámica. 


\section{CARACTERÍSTICAS DE LOS SISTEMAS}

Según Herrscher (2008: 166) dentro del campo de aplicación del enfoque de sistemas, se pueden identificar tres grupos diferentes de "solucionadores de problemas" a partir de tres distintas concepciones de sistemas:

a) Los sistemas duros.- Aquellos en los que el problema está definido, el objetivo está dado y es cuestión de alcanzarlo lo más eficiente posible.

b) Los sistemas blandos.- Aquellos en que el primer problema es definir el problema, varios actores tienen diversos objetivos y aparecen factores sociales complejos y ambiguos.

c) Los sistemas críticos.- También conocidos como sistemas emancipatorios. Son aquellos en los que dichos factores sociales, se encaran al servicio del cambio social a través de lo prescriptivo y aún de la intervención activa, para solucionar los graves problemas de la sociedad (marginación, violencia o destrucción de la naturaleza).

Esta clasificación, es un panorama general en el cuál se puede identificar un sistema u otro, sin embargo, esta diferencia no excluye la posible acción de compartir características, metodologías y herramientas. Es decir, la clasificación está dada a partir del panorama del punto de arranque en el que se pretende intervenir en determinado momento en un sistema social dado.

Las características que están presentes en los sistemas (sin importar el grupo conceptual al que pertenezcan) son los siguientes según Herrscher (2008: 168-188):

a) Interdependencia.- La define como el "dar más importancia a la vinculación entre las partes que a las partes en sí", "reconocer que la alteración de uno de los vínculos, digamos entre $\mathrm{A}$ y $\mathrm{B}$, afectará de algún modo a los vínculos entre $\mathrm{A}$ y $\mathrm{C}$, entre $\mathrm{B}$ y $\mathrm{D}$, entre $\mathrm{C}$ y $\mathrm{D}$, etc." y "comprender por lo tanto que, así como el todo vive a través de sus partes, las partes sólo viven en función del todo". Asimismo el autor indica que "no se trata de decir que la parte carece de importancia ni que, por pertenecer al todo, deba perder su identidad o sus objetivos propios (eso sí: no contrarios a los del todo)”.

b) Interacción.- Es la interdependencia (una propiedad latente) llevada a la práctica (un comportamiento efectivo) en el cuál, ambas puntas de un vínculo "partes-todo" sacrifican algo: la parte, una porción de autonomía. Y el todo, una porción de su autoridad jerárquica. 
c) Diversidad.- Lo establece como la circunstancia de que un conjunto esté compuesto por elementos diferentes por causa de decisión basada en criterios o preferencias.

d) Recursividad.- Bertoldo, 1985 (en François, 1997: 292) define a la recursividad como "la aplicación de una función a su propio valor para generar una serie de valores similares". En ella intervienen tres características: el carácter no lineal de muchas relaciones, el carácter bidimensional de muchos vínculos y el carácter fractal de muchos subsistemas.

e) Dinámica.- Herrscher (2008: 168) la considera como "la más poderosa herramienta que ha producido el enfoque de sistemas". Destaca su valor como "descriptor, descubridor y resolvedor de problemas" y sus límites por "ser no más, pero tampoco menos que una herramienta". Asimismo, su lógica está basada en la retroalimentación (positiva o negativa) a partir del encadenamiento "control=aprendizaje=retroalimentación".

f) Autorreferencia.- Para Foerster (en Herrscher, 2008: 34) es la propiedad de los sistemas de volver sobre sí mismos, de "morderse la cola", de definirse y reinventarse continuamente, representa una importantísima cualidad del planeamiento. En sus palabras "Planificar y continuamente re-planificar el planeamiento".

\section{LOS SISTEMAS COMPLEJOS EN LAS ORGANIZACIONES SOCIALES}

De acuerdo con Van Gigch (2001: 15) los sistemas revelan que comparten una característica: la complejidad. Del latín complexus, referido a lo que está tejido en conjunto. La complejidad es el resultado de la multiplicidad y embrollo de la interacción del hombre en los sistemas.

Por otra parte, Etkin (2007: 34) concibe a lo complejo en el admitir la coexistencia de orden y desorden, de obligación y libertad, sin ver en ello un drama por lo que no debería tratarse como un sinónimo de negatividad o desigualdad, ya que ello también puede llevar a cabo el reforzamiento de las relaciones y a construir formas de superar problemas no previstos en un sentido constructivo.

Según Etkin (2007: 358-359) la complejidad en la realidad organizacional se identifican tres aspectos coexistentes cotidianos: el diseño (de estructuras y procesos formales), las actividades emergentes (no pensadas) y la divergencia de ideas. Esta característica de complejidad en los sistemas organizacionales, hace que sus componentes revelen cambios inciertos y de difícil predicción, sin embargo; lejos de ser estos factores negativos, pueden percibirse como evidencias de errores, para posteriormente ofrecer formas de superarlos. Con ello se buscaría una mejora para el sistema llamado desorden creativo. 
Respecto a lo anterior, Morin (1994: 81) destaca en su análisis de la complejidad que "las relaciones al interior de una organización de una sociedad o una empresa son complementarias y antagonistas al mismo tiempo". Asume así, que este hecho podría generar entre los miembros una creatividad no planificada.

Sin embargo, cuando nos referimos a la complejidad relacionada a los sistemas sociales, es fundamental referirnos a la teoría de Niklas Luhmann, autor que nos ofrece una forma de comprender a la sociedad, sus relaciones y entorno a partir de los sistemas sociales complejos. Respecto a la forma de análisis de los sistemas sociales Luhmann asume lo siguiente:

..."Un sistema social, es distinto de su entorno y operacionalmente cerrado, o sea, capaz de producir por sí mismo, elementos propios y estructuras propias. Esta definición general es necesaria pero insuficiente para el análisis de los sistemas sociales. Para la observación de los sistemas sociales, es necesario distinguirlos de sistemas de otro tipo (mecánicos, vivos, psíquicos) con el objeto de evitar confusiones analíticas y evitar de igual forma, considerar lo idéntico en un plano, como idéntico también en los otros"... (Luhmann, 1998: 27).

La siguiente figura (1) muestra la distinción de los tres planos de análisis (mencionados en el párrafo anterior) que permiten diferenciar a los sistemas sociales de otros tipos de sistemas y sus relaciones entre sí.

Figura 1.- Los Sistemas Sociales.

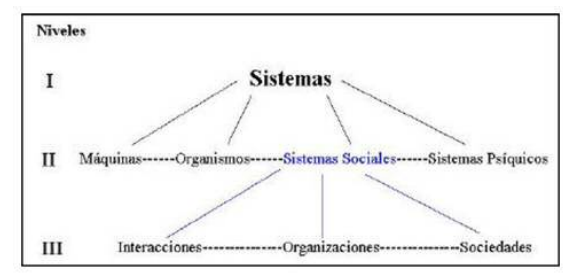

Fuente: Luhmann (1998: 27).

Como se aprecia en el esquema anterior, los sistemas sociales están sujetos a interacciones, organizaciones y sociedades que por el hecho de formar parte en un mismo tiempo y espacio, comparten la característica de complejidad. (Luhmann, 1998: 47-48) afirma que en ..."la complejidad es un estado de cosas autocondicionado, debido a que los elementos deben constituirse complejamente para fungir como unidad en el nivel superior de la formación de sistemas, y por lo tanto su capacidad de acoplamiento es limitada. De ahí que la complejidad se reproduzca como un hecho inevitable en cualquier nivel superior 
de la formación de sistemas. Esta autorreferencia de la complejidad será después "internalizada" por el sistema como autorreferencia"...

..."Asimismo, se puede hablar de complejidad superior o complejidad inferior (diferencia de complejidad o gradiente de complejidad) siempre y cuando, en ambos sentidos, esté supuesta una complejidad menor. Este es el caso de la relación entre sistema y entorno. En cambio, se debería hablar de reducción de complejidad, en un sentido más restringido, cuando el complejo de relaciones de un entramado complejo es reconstruido mediante un segundo entramado de relaciones menores. Sólo la complejidad puede reducir complejidad"... (Luhmann, 1998: 49).

\section{LA FUNCIONALIDAD, EFECTIVIDAD Y SINERGIA EN LOS SISTEMAS COMPLEJOS}

La funcionalidad, efectividad y sinergia son propiedades que idealmente deberían tomar lugar dentro de los sistemas complejos. A veces pueden encontrarse en diferentes escalas, es decir, en un sistema puede existir la funcionalidad y en otros puede existir la funcionalidad y la efectividad, sin embargo, lo mejor es poder alcanzar estas tres propiedades en un mismo sistema o por lo menos procurar la existencia de por lo menos una de estas. Es importante mencionar que estas propiedades funcionan en serie, siendo la sinergia la más difícil de alcanzar y permanecer. A continuación se abordará la importancia de este idealismo.

La funcionalidad se puede aplicar a alguna cosa que está pensada y creada para tener una utilidad práctica, y no estética (IPN, 2015: 40). De acuerdo con Bouza (2000: 53) la efectividad es algo que conduce a un efecto o una consecuencia real verdadera y que no deja lugar a duda del hecho. Asimismo, la efectividad del sistema, va a estar dada conforme se cumplen los objetivos de calidad que se propone al implantar el sistema, sus resultados bajo las condiciones reales. Los cuales se pueden expresar como:

Efectividad $=$ Es la relación de objetivos/ resultados bajo condiciones reales

En el mismo sentido, la Secretaría de Turismo (SECTUR, 2008: 14) establece que la "eficacia" se puede establecer en términos del grado de cumplimiento de los objetivos que motivaron la intervención y se concentra exclusivamente en el análisis de los efectos positivos que fueron previstos en el diseño de algún programa y en los objetivos de la intervención. Así, existen dos enfoques para evaluar la eficiencia de un programa social:

- El análisis costo- beneficio.- Expresa los beneficios en términos monetarios.

- El análisis costo-efectividad.- Los beneficios son valorados a través de indicadores que 
miden los cambios en las condiciones de bienestar.

Por otra parte, se puede decir que la palabra sinergia proviene de la antigua cultura griega y su traducción literal sería cooperación; no obstante (según la Real Academia Española, 2012) el término sinergia se refiere a la acción de dos (o más) causas cuyo efecto es superior a la suma de los efectos individuales. La sinergia es también, la integración de sistemas que conforman un nuevo objeto. Por ello, cuando dos o más elementos se unen sinérgicamente crean un resultado que aprovecha y maximiza las cualidades de cada uno de los elementos.

Johansen (1993: 35-36) integra la importancia de la sinergia en la teoría general de sistemas, menciona que cuando la suma de las partes es diferente del todo decimos que posee o existe sinergia y la explica citando a Fuller: "un objeto posee sinergia cuando el examen de una o alguna de sus partes (incluso a cada una de sus partes) en forma aislada, no puede explicar o predecir la conducta del todo".

Ligado a este concepto, se encuentra la recursividad, que señala que un sistema sinérgico está compuesto a su vez de subsistemas que también son sinérgicos, como establece este autor, "el todo es más que la suma de las partes".

A partir de las anteriores definiciones, se puede establecer la importancia de la existencia de los tres elementos (funcionalidad, efectividad y sinergia) en los sistemas complejos, ya que brindan la oportunidad de incrementar efectos positivos, principalmente por:

- La identificación e integración de todos los elementos o componentes necesarios para su funcionamiento.

- La seguridad de que con el funcionamiento que se lleva a cabo es suficiente también para poder llegar a los objetivos y metas establecidas, así como el permitir la autoevaluación y retroalimentación para poder identificar oportunidades y amenazas con el fin siempre de mejorar dicho funcionamiento.

- La posibilidad de que con el buen funcionamiento y eficacia se puedan crear fenómenos positivos más allá de lo previsto; los cuales brinden otras posibilidades de éxito que van más allá de las ya establecidas anteriormente así como el surgimiento de elementos difíciles de alcanzar como el capital social y valores entre los actores que integran dicho sistema.

Sin embargo; estos elementos son difíciles de alcanzar si no se lleva a cabo un proceso de planeación que permita en cada etapa vincularlas y mantenerlas dentro de la visión general del sistema. 


\section{LA PLANEACIÓN SISTÉMICA}

Cuando se pretende intervenir dentro un sistema, es importante orientarnos hacia la planeación como herramienta fundamental para su análisis y comprensión. La planeación sistémica, es una de las diferentes clasificaciones de la planeación y la que más puede ser de utilidad en el mundo de los sistemas complejos.

Ordaz y Saldaña (2005: 83) subdivide claramente once tipos de planeación con base en sus características propias para poder aplicarlas en espacios regionales. Entre los tipos que presenta, se encuentra la planeación sistémica. Dentro de la planeación sistémica, Guerra (1977 en Ordaz y Saldaña, 2005: 83) menciona que las relaciones del hombre con la sociedad y el entorno pueden ser identificadas en términos de sistemas porque contiene partes relacionadas entre sí, y en algún sentido constituye un todo completo. En este tipo de planeación se intenta definir el sistema identificando las partes o elementos por un lado, y por otro las conexiones o interacciones.

La diferencia entre el planeamiento y planeamiento sistémico puede encontrarse dentro de sus objetivos. Los objetivos del planeamiento son múltiples y van desde lo más "blando" e idealizado hasta lo más "duro" y concreto ya que permite (Herrscher, 2008: 224):
- $\quad$ Pensar en el futuro para preverlo
- Definir objetivos y el modo de alcanzarlos
- Dar forma e integrar conjuntos de estrategias o proyectos
- Programar el uso de recursos y establecer pautas de gestión y resultados
- Decidir por anticipado qué y cómo debe hacerse algo.

En cambio, cuando nos referimos al planeamiento sistémico, se puede encontrar además un estructura (aunque más compleja) visiblemente más viable para en su proceso que permite a los participantes tener una idea más clara sobre cómo, cuándo y porqué abordar una situación determinada. A continuación se presenta el modelo del diseño sistémico de Gharajedaghi (en Herrscher, 2008: 238) en donde se puede observar la integración de los diversos sistemas internos y externos en las organizaciones como ejemplo del proceso de planeamiento sistémico (Figura 2). 
Figura 2. Diseño Sistémico del Proceso de Planeamiento.

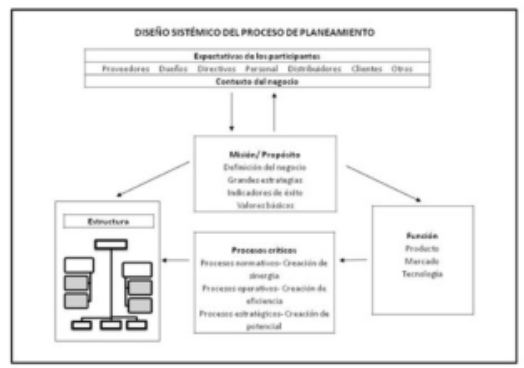

Fuente: Gharajedaghi (1999 en Herrscher, 2008: 238).

Otro modelo que permite diferenciar al planeamiento del planeamiento sistémico es el que presenta Schwaninger (en Herrscher 2008: 240) quien por medio de tres niveles conductivos expresa el proceso de planeamiento sistémico (figura 3).

Figura 3. Los tres niveles de la conducción del planeamiento sistémico.

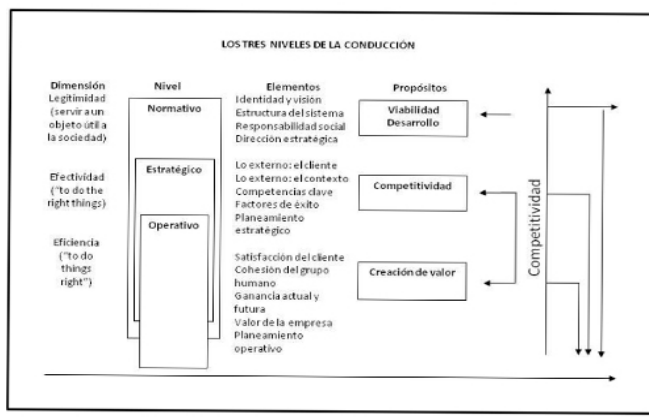

Fuente: Schwaninger 2003 (en Herrscher 2008: 240).

Cuando se planifica con una visión sistémica, se puede planificar también hacia un enfoque sustentable, ya que ambas (la visión sistémica y el desarrollo sustentable) cuentan con características y propiedades que pueden ser compatibles para ser funcionales, efectivas y sinérgicas en su correcta aplicación dentro de un espacio. 


\section{LA VISIÓN SISTÉMICA Y SU IMPORTANCIA PARA LOS ESTUDIOS CON ENFOQUE SUSTENTABLE: EL TURISMO COMO EJEMPLO.}

La relación entre la visión sistémica y el desarrollo sustentable es muy estrecha. Particularmente, cuando se habla de visión sistémica, se refiere a la visión del todo para comprender las partes, del análisis de lo global para entender lo particular, de propiedades emergentes y retroalimentación, de entropía y organización, de búsqueda de funcionalidad y eficacia, de la esencia de los sistemas complejos, de compartimiento de los seres humanos y de los sistemas sociales.

Por otra parte y de acuerdo con Antequera (2005: 168) la sostenibilidad no se refiere sólo al mantenimiento de la organización de los sistemas naturales que soportan el desarrollo del sistema socio-cultural humano sobre el planeta sino que trata a la luz de la preservación en el tiempo de los capitales culturales, institucionales, productivos, etc., que posibilitan el desarrollo humano y social.

Estas pautas conductuales del desarrollo sustentable son principios que debe tener la sociedad para poder mantenerse en el tiempo. Así, la visión sistémica de la realidad, puede contribuir en el análisis de la sustentabilidad local.

El desarrollo sustentable integra tanto en su concepto como en su aplicación, propiedades que comparten los sistemas, es decir, nos enfrentamos a un fenómeno, multidisciplinario, interdisciplinario, autoorganizado, cambiante en tiempo y espacio y que además comparte relaciones estrechas e interacciones entre sus componentes que en conjunto, son determinantes en el comportamiento del mismo. Estas características son también lo que se denomina como sistema complejo.

Siguiendo con la idea anterior, la noción de entender al desarrollo sustentable desde un enfoque sistémico, permite explicar la complejidad del conocimiento de la realidad y la constante ampliación de la información. El problema esencial es que cuanto más complejo es un sistema, más depende su comportamiento general de las interacciones entre diferentes elementos y más difícil resulta comprender o modelar éstos en el momento de referencia de las disciplinas tradicionales (González, 2002: 96).

De acuerdo con Campos (2010: 212) las ciencias sociales han utilizado la teoría de sistemas para tratar de reconocer procesos complejos donde las sociedades, los territorios o las economías articulan partes que sumadas configuran una totalidad funcional. En el caso del turismo, permite comprender la realidad ambiental, territorial, social y económica a través de su comportamiento sistémico y analizar de manera fluida y compleja, la 
articulación dinámica de las partes que componen los sistemas sociales, territoriales y económicos.

Siguiendo con la idea de los autores antes mencionados dentro de la Teoría General de Sistemas, se puede asumir que el turismo representa a una organización compleja integrada por elementos sociales, la cual debe visualizarse como un sistema que permita identificar relaciones, sus componentes y características propias, permitiendo así la oportunidad de crear sinergias en su desarrollo, minimizar y prevenir posibles impactos negativos que pudiera generar y aprovechar adecuadamente los impactos positivos posibles. Lo anterior a través de un modelo que permita en gran medida ser un dirigente para los actores involucrados dentro la planificación en la actividad turística.

\section{EL MODELO EN LOS SISTEMAS Y SU APLICACIÓN EN EL TURISMO}

El término "modelo" es referido como la representación esquematizada del sistema que recoge los principales elementos y las interrelaciones más significativas (Pujadas y Font, 1998: 39). De acuerdo con Herrscher (2008: 272) todos los modelos sistémicos integran, relacionan y aclaran.

A pesar de que un modelo puede percibirse como un esquema o mapa que nos proporciona una dirección y nos brinda la suficiente información sobre cómo controlar nuestro sistema, los modelos son limitados en cuánto a indicar qué camino seguir, en resolver problemas por sí solos y sobre todo ninguno puede tener un resultado asegurado. Es decir; por su naturaleza, los modelos pueden simplificar y apoyar en el control de un escenario o situación determinada, pero necesitan complementarse con herramientas diversas para intentar alcanzar los resultados, los cuáles por el hecho de "integrar un modelo u otro" no significa que sean los esperados.

Al respecto, Herrscher (2008: 161) nos recuerda la expresión clásica que "los modelos constituyen una de las herramientas más maravillosas que hay, con tal de que no se crea en ellos", dejando en claro que el modelo cómo expresión simplificada, es insustituible para comprender una realidad compleja y saber actuar frente a ella y por otra parte puntualiza que los modelos no son la realidad, lo cual advierte el peligro de "administrar el modelo y no la realidad misma".

En el turismo (desde un enfoque sistémico) los modelos buscan expresar los elementos que forman parte el sistema turístico, sus interrelaciones y su relación con el ambiente externo. Se considera que el abordaje sistemático es un paradigma en los estudios turísticos porque la visión sistemática tiene gran difusión, alcance y utilización en los estudios del área y hasta la actualidad es la teoría que mejor explica la dinámica del turismo a pesar de que aún contiene elementos que dificultan la comprensión (Panosso, 
2007: 396).

Entre los autores que han integrado en sus investigaciones hacia un enfoque sistémico, se encuentra el modelo de Leiper (1979: 404) que presenta una forma simple y amplia al representar el turismo, constituyéndose por elementos e interrelaciones reconocidas como fundamentales. En 1990, Cooper adopta el modelo de Leiper que sirve de base conceptual al considerar, las formas de relación entre los turistas, elementos geográficos y la industria turística. Corroborando estas afirmaciones Cooper et al. (1997: 40) describen la aportación de Leiper:

..."Tiene la capacidad de incorporar al turismo visiones interdisciplinarias porque no está enraizado en cualquier tema o disciplina en particular, sino que provee una estructura dentro de la cual se pueden situar los puntos de vista de las distintas disciplinas. Se la puede usar en cualquier escala o nivel de generalización (desde un resort hasta en la industria internacional). El modelo es infinitamente flexible, permite la incorporación de formas diferentes de turismo, y al mismo tiempo demuestra sus elementos comunes"...

En el caso de Cooper, en 1990 retoma este modelo y los integra como una de las bases de sus obras. Además, realiza obras que abarcan el estudio de sistemas complejos, intentando proveer herramientas y estudios de casos generales en los que puedan adaptarse a los diferentes modelos que integra en sus libros.

En este sentido, se conocen alrededor de 200 modelos para explicar el turismo como un sistema en las últimas cuatro décadas. De acuerdo con Barretto (2007: 10) solamente en lengua inglesa Getz (1986: 24) identificó más de ciento cincuenta, que subdividió en teóricos, de planeamiento y gestión, y predictivos divididos en teóricos en totales, espacio-temporales, motivacionales, de impacto general, de impacto económico, de impacto socio cultural y de impacto ecológico. Asimismo, los modelos sistémicos de planeamiento y gestión fueron subdivididos por este autor en modelos de desarrollo de área, desarrollo de proyectos, gestión y marketing, y modelo conceptual. Los de predicción fueron subdivididos en econométricos, temporales y físicos.

Otros modelos de autores importantes son Jafari y McIntosh en 1990, Smith en 1989 y Boniface en 1987 (citados por Cooper et al., 1997) quienes comparten la idea de crear bibliografía básica de estudios avanzados sobre turismo.

En otro contexto, si se percibe a la actividad turística como un sistema social complejo y éste se pretende representar a través de un modelo, es pertinente integrar la sinergia, funcionalidad y efectividad como elementos ideales dentro de su planificación procurando que estén presentes como parte fundamental en su desarrollo. Lo anterior debido a la relevancia que representa en el éxito o colapso dentro de un sistema. 


\section{LA PLANIFICACIÓN TURÍSTICA DESDE UN ENFOQUE SUSTENTABLE}

Para la SECTUR (2004: 21) pensar a futuro es la base de la planeación turística, ya que considera que el único modo de sustentar una economía a largo plazo basada en el turismo, es administrar los atractivos con criterios de sustentabilidad para evitar su agotamiento, ya que la sobreexplotación lleva en sí misma al fracaso a corto plazo. Sin embargo, como ya se ha mencionado anteriormente, el desarrollo sustentable sigue siendo en la mayoría de los casos un intento de un desarrollo respetuoso tanto del entorno como en temporalidad, que derivado de esa complejidad, no se tienen parámetros específicos a seguir para llegar a ella.

Según la SECTUR (2004: 21) el significado del Turismo Sustentable proviene del concepto "Desarrollo Sustentable". La Organización Mundial del Turismo (OMT, 1999: 22) establece al Desarrollo Sostenible del Turismo como la actividad que atiende a las necesidades de los turistas actuales y de las regiones receptoras, y al mismo tiempo protege y fomenta las oportunidades para el futuro. Asimismo, lo concibe como una guía hacia la gestión de todos los recursos, de forma que puedan satisfacer las necesidades económicas, sociales y estéticas, respetando al mismo tiempo la integración cultural, los procesos ecológicos esenciales, la diversidad biológica y los sistemas que sostienen la vida.

Entre las diversas definiciones que se le pueden asignar al turismo sostenible o sustentable, la que más es utilizada debido al grado jerárquico como regulador de la actividad es precisamente el que establece la OMT (2001 en Carrión, 2014): "Capacidad de un destino de permanecer competitivo en relación con otros destinos más nuevos y menos explotados; de atraer visitantes de primera vez, así como visitas repetidas; de conservarse culturalmente singular y de estar en equilibrio con el medio ambiente".

Siguiendo con los criterios que establece la OMT sobre el tema, establece que las directrices para el desarrollo sostenible del turismo y las prácticas de gestión sostenible son aplicables a todas las formas de turismo en todos los destinos, incluidos el turismo de masa y los diversos segmentos turísticos. Los principios de sostenibilidad se refieren a los aspectos ambiental, económico y sociocultural del desarrollo turístico, habiéndose de establecer un equilibrio adecuado entre estas tres dimensiones para garantizar su sostenibilidad a largo plazo.

Asimismo establece que el desarrollo sostenible del turismo exige la participación informada de todos los agentes relevantes, así como un liderazgo político firme para lograr una colaboración amplia y establecer un consenso; ya que para lograrlo, se requiere de un proceso continuo y un monitoreo constante de los impactos para introducir las 
medidas preventivas o correctivas que resulten necesarias.

En el mismo sentido, el crecimiento constante de ésta industria y su tendencia consiguiente a la masificación se produce de forma paralela con una mayor conciencia ambiental de unos consumidores que cada vez exigirán destinos turísticos más limpios, seguros y benévolos para el medio ambiente. Estas dos tendencias, convierten en algo imperativo el desarrollo y la gestión de una industria turística sostenible (OMT, 1999: 3).

Por ello la OMT (1999: 9) reitera que estos segmentos del turismo presentarán factores de cambio en este proceso de escala global. Algunas señales de estos cambios son:

- La globalización de la economía turística.

- El acortamiento de las distancias.

- Nuevos modos de transmitir y gestionar la información y la promoción turística.

- La competitividad en todas las escalas de destino.

- La sostenibilidad ambiental y la autenticidad cultural como nuevos factores claves de la agenda turística.

Lo anterior apunta a lo que Ascanio y Vinicius (2009: 114) asumen de la actividad turística: "el turismo del siglo XXI debe asegurar experiencias innovadoras, al mismo tiempo que ofrecer seguridad y destinos muy particulares que puedan satisfacer las visitas".

Estas experiencias innovadoras requieren de un cambio en la racionalidad humana respecto a las actuales formas de uso de los recursos naturales para lograr su persistencia en el tiempo y espacio y que es más viable "planear hacia las vías o trayectorias que puedan llevar a la sustentabilidad del sistema", más que planear hacia el concepto per se (SECTUR, 2007: 26). Este nuevo modelo de turismo se encamina al reequilibrio de componentes económicos, sociales, ambientales y culturales que intervienen en su desarrollo, lo cual requerirá como punto de partida la objetiva reflexión y aceptación de las teorías fundamentales de la sustentabilidad del turismo y su planificación.

En este sentido, la planificación juega un papel importante dentro del enfoque sustentable. Desde el análisis proveniente de su conceptualización, se pueden observar aspectos que sólo pueden alcanzarse con el apoyo de una herramienta que comparta una visión integral, compatible y cíclica entre otras características, las cuales pueden considerarse como requisitos que cumple la planificación.

Al respecto, Salinas (2005) reitera (a partir de la corriente del Desarrollo Sustentable) que en la planificación del desarrollo se deben tomar cinco dimensiones básicas de la sustentabilidad como se muestra en la figura 4. 
Figura 4.- Dimensiones básicas de la sustentabilidad como parte de la planificación.

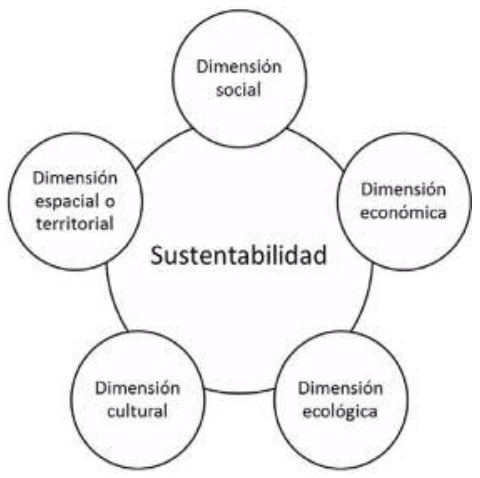

Fuente: Elaboración propia con datos de Salinas (2005).

Como se observa en la figura 4, la sustentabilidad requiere de la integración de componentes estrechamente relacionados pero que al mismo tiempo son caracterizados por si solos como escenarios complejos lo cual dificulta esta tarea de integración. Por tal motivo, el desarrollo sustentable establece la necesidad de un nuevo tipo de planificación que permita acercar el conocimiento hacia acciones, por lo que este modelo de planificación debe ser según Friedmann (1992: 87) normativo, innovador, político, negociador y basado en el aprendizaje social. Este tipo de modelo es conocido como planificación sustentable, ambiental, ecológica, espacial o estratégica; el cual puede identificarse a través del siguiente concepto:

..."El instrumento dirigido a planear y programar el uso del territorio, las actividades productivas, la organización de los asentamientos humanos y el desarrollo de la sociedad, en congruencia con el potencial natural de la tierra, el aprovechamiento sustentable de los recursos naturales y humanos y la protección y calidad del medio ambiente" (Salinas, 2005)...

De acuerdo con Diegues (1992 en Negrão, 2000: 16-17) la deficiencia de la sustentabilidad no es el concepto, sino el instrumental de la economía, que intenta resolver los problemas del desarrollo sustentable de manera aislada. Instrumentos un tanto generales (en la mayoría de los casos) que direccionan a los actores turísticos a diversas alternativas para adoptar en una situación u otra. Por lo anterior mencionado, en este apartado se identificaran algunos modelos e instrumentos que son relevantes dentro de la planificación turística sustentable. 
Como primer acercamiento a la planificación turística sustentable es importante retomar las siguientes distinciones en palabras de Acerenza (2006: 11): ..."En la política del desarrollo sostenible deben distinguirse dos dimensiones interrelacionadas; por un lado, la política general de desarrollo sostenible del turismo y, por otro, las políticas turísticas específicas, destinadas a corregir el deterioro del medio ambiente ocasionado por el turismo masivo de alto impacto, y el desarrollo de los distintos tipos y modalidades de turismo, con los correspondientes apoyos para fomentarlos. La conjunción de estas dos políticas conforman lo que llamaremos política integral de desarrollo sostenible del turismo"...

Siguiendo con aclaraciones relevantes del turismo sustentable y su planificación es importante resaltar también las palabras de Ascanio (2005): ..."El turismo sustentable basado en la presencia de la comunidad organizada protectora de su hábitat es una cosa, y otra es el turismo sustentable basado en las buenas prácticas ambientales para ahorrar agua, gestión de residuos sólidos y otras acciones similares que puedan preservar los recursos del medio a través de ecoetiquetas y códigos de conducta, pero que también pueden producir un incremento del turismo masivo"...

Por lo anterior mencionado la gestión juega un papel importante dentro de la planificación turística sustentable. Al respecto, Vignati (2009: 57) construye cuatro conceptos fundamentales en la gestión del turismo:

1.- Sostenibilidad económica del turismo.- Capacidad de las empresas turísticas para ofrecer servicios de calidad que produzcan ganancias superiores a los costos.

2.- Sostenibilidad ambiental del turismo.- Nivel arriba del cual se producen impactos ambientales que perjudican la calidad del ambiente y de la oferta turística.

3.- Sostenibilidad social del turismo.- Nivel de tolerancia de la sociedad ante la presencia de turistas y del desarrollo turístico en general.

4.- Sostenibilidad del turista.- Nivel determinado por la capacidad de satisfacción del turista ante la cantidad de personas que visitan a la vez un mismo destino turístico.

Estos cuatro conceptos son niveles de tolerancia que tienen los involucrados directos e indirectos de la actividad turística los cuales deben ser abordados como un conjunto de componentes dentro de un sistema general en el que se puedan identificar las relaciones, características y demás propiedades del sistema que puedan acercar a cada involucrado al equilibrio a través del tiempo. 
Para planificar con enfoque sustentable podemos incluir a Vera et al. (2001: 11) quienes presentan un modelo de desarrollo turístico sostenible a partir de tres ejes básicos como se muestra en la figura 5.

Figura 5.- Ejes básicos del Turismo sostenible.

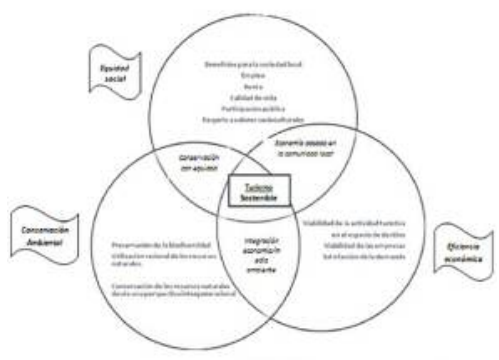

Fuente: Adaptado de Vera et al. (2001:11).

A partir del modelo los autores mencionan que "reconocidos los ejes básicos del desarrollo turístico sostenible, parece claro que la sustentabilidad no puede identificarse con un estado ideal, prácticamente utópico. Debe concebirse más bien, como un proceso de cambio cualitativo de acuerdo con los postulados de la Comisión Mundial sobre Medio Ambiente y Desarrollo en 1988 que oriente, o reoriente, el desarrollo turístico hacia objetivos alcanzables".

Desde el punto de vista territorial Vera y Cols (1997: 406 en Ascanio y Vinicius, 2009: 25) mencionan que "las formas y modelos de organización del territorio previamente al desarrollo de las actividades turísticas, han condicionado en buena medida los resultados que se pueden obtener con posterioridad y justifican de la misma manera las modalidades de intersección territorial".

En otro contexto, los indicadores sustentables tienen gran importancia dentro de la planificación sustentable y por lo consecuente en la planificación del turismo sustentable. Al respecto, Bermejo y Nebreda (1998: 10-14) asumen que el desarrollo sostenible exige indicadores adaptados al carácter integral y multidimensional de los procesos de desarrollo, aunque este objetivo implique mayor complejidad en su diseño, obtención e interpretación. Así, la Agenda 21 (SECTUR, 2008) es un ejemplo claro de esta exigencia, la cual ha direccionado a las Administraciones Públicas para que desarrollen indicadores de sostenibilidad, por lo menos en el plano de la definición teórica. Estos indicadores, también son implementados como objeto de estudio dentro de la planificación del turismo sustentable. 
De acuerdo con Vera et al. (2001: 15) los indicadores de sostenibilidad deben recoger las tres dimensiones propias de este paradigma (económica, ambiental y sociocultural) y por tanto no deben circunscribirse exclusivamente a indicadores de tipo ambiental, a pesar de que los avances más significativos se produzcan en este campo.

Entre las diversas organizaciones internacionales, nacionales y organizaciones no gubernamentales que han emprendido iniciativas relacionadas con los indicadores de sostenibilidad desde diversos enfoques se encuentran la Organización para la Cooperación y el Desarrollo Económico (OECD, 1993), la Agencia Europea de Medio Ambiente (1998), la Organización Mundial del Turismo (OMT,2005) entre otras; así como los trabajos e investigaciones del Institut Francais de'1 Environnement (2000), los National Sustainable Tourism Indicators, los indicadores de integración del turismo y el medio ambiente de Portugal (Ministerio do Ambiente, 1998) y el Sistema Español de Indicadores Ambientales (Ministerio de Medio Ambiente, 2003) por mencionar algunos.

\section{CONCLUSIONES}

El comportamiento sistémico en términos de sinergia, funcionalidad y efectividad de la actividad turística de un destino puede medirse con información de indicadores sobre los componentes actores del turismo, desarrollo urbano, entorno socioeconómico, medio ambiente y turismo. Sin embargo, la calidad del monitoreo del comportamiento sistémico dependerá de la calidad de la información de los indicadores en el momento de realizar el diagnóstico.

Los componentes del sistema turístico sí pueden integrarse a partir de un enfoque sistémico permitiendo desarrollar la actividad turística bajo un enfoque sustentable debido a sus características como la complejidad, dinámica e interdisciplinaridad, entre otros, lo que permite la adaptación de los principios de sustentabilidad. Es decir, comparten propiedades comunes y que pesar de ser disciplinas diferentes, el enfoque sistémico permite explicar la dinámica del turismo pese a la dificultad en la comprensión de los elementos cuando se le atribuyen los principios sustentables.

Este enfoque, permitió vislumbrar al turismo como un sistema complejo integrado por componentes estrechamente relacionados con el medio ambiente y la sociedad, que deben ser considerados para entender la importancia de la autoorganización, multidisciplinaridad, dinámica y evolución, determinantes de la sinergia entre los componentes de un sistema turístico sustentable. 


\section{BIBLIOGRAFÍA}

Acerenza, M. (2006). "Necesidad de precisar el contenido y alcance de la política de desarrollo sostenible del turismo". Aportes y transferencias, 10(1): 11-21.

Agencia Europea de Medio Ambiente (1998). Europe's Environment: The Second Assessment. Copenhagen: Elsevier Science Ltd.

Antequera, J. (2005). El Potencial de Sostenibilidad de Asentamientos Humanos. [En línea]. [Fecha de consulta 18 de abril de 2015]. Disponible en http://www.eumed.net/ libros/2005/ja-sost/

Ascanio, A. (2005). "Turismo, la naturaleza y las comunidades: nuevas maneras de parcelar el territorio". Os Urbanitas [En línea], 2(2) [fecha de consulta 26 de mayo de 2015]. Disponible en http://www.osurbanitas.org/osurbanitas2/ascanio2005.html.

Ascanio, A. y Vinicius, M. (2009). Turismo Sustentable: el equilibrio necesario en el siglo XXI. México: Trillas.

Ascanio, A. y Vinicius, M. (2009). Turismo Sustentable: el equilibrio necesario en el siglo XXI. México: Trillas.

Barretto, M. (2007). Turismo y cultura. Relaciones, contradicciones y expectativas. España: Colección PASOS.

Bermejo, R. y Nebreda, A. (1998). "Conceptos e instrumentos para la sostenibilidad local". Cuadernos Bakeaz, 26: 1-18.

Bertalanffy, L. (1992). Perspectivas en la teoría general de sistemas. Madrid: Alianza Universidad.

Boulding, K. (1964). General systems as a point of view, in J. Mesarovic (ed).Views on general systems theory. New York: John Wiley.

Bouza, A. (2000). "Reflexiones acerca del uso de los conceptos de eficiencia, eficacia y efectividad en el sector de la salud". Revista Cubana Salud Pública, 26(1): 50-56.

Campos, B. (2010). Complejidad y territorialidad en los espacios turísticos. México: Plaza y Valdés.

Carrión, N. (2014). Propuesta de la implementación del complejo turístico "las tres chorreras" en la parroquia "el ingenio" perteneciente al Cantón "Marcabelí", Provincia de "el Oro". Tesis (Licenciatura en Gestión y Desarrollo Turístico. Cuenca, Ecuador: Universidad del Azuay. Facultad de Filosofía. Escuela de Turismo. 61p.

Comisión Mundial del Medio Ambiente y del Desarrollo (1988). Nuestro Futuro Común. Madrid: Alianza.

Cooper, C., Fletcher, J. y Wanhill, S. (1997). Turismo: Principios y práctica. México: Diana.

Etkin, J. (2007). Capital social y valores en la organización sustentable. Buenos Aires: Granica.

Foerster, H. (1949). Cibernética: Transacciones de la sexta conferencia. Nueva York: Fundación.

François, C. (1997). International Encyclopedia of Systems and Cybernetics. Munich: 
Saur.

Friedmann, J. (1992). "Planificación para el siglo XXI: El desafío del posmodernismo". EURE, 18(55): 79-89.

Gerard, R. (1956). "Evolución biológica y cultural: Algunas analogías y exploraciones". Ciencia del comportamiento, 1: 6-34.

Getz, D. (1986). "Models in tourism planning: Towards integration of theory and practice". Tourism Management, 1(7): 21-32.

Gigch Van, J. (2001). Teoría General de Sistemas. México: Trillas.

González, M. (2002). La Ciudad Sostenible. Planificación y Teoría de Sistemas. Boletín de la A.G.E. No. 33 Departamento de Geografía. Universidad de León. España.

Gunther, T. y Gómez-Jara, C. (2005). El derecho como sistema autopoiético de la sociedad global. Perú: Centro de Investigación en Filosofía y Derecho.

Herrscher, E. (2008). Planeamiento Sistémico. Un enfoque estratégico en la turbulencia. Buenos Aires: Granica.

IFEN (2000). Les Indicateurs. Tourisme, environnement, territories. Orleáns: IFEN.

IPN (2015). Programa integral de fortalecimiento de la infraestructura física educativa en el IPN. México: IPN.

Johansen O. (1993). Introducción a la teoría general de sistemas. México: LIMUSA Leiper, N. (1979). "The Framework of Tourism: Towards a Definition of Tourism, Tourist, and the Tourist Industry". Annals of Tourism Research, 6(4): 390-407.

Luhmann N. (1998). Teoría de los Sistemas Sociales. México: Universidad Iberoamericana.

Luhmann, N. (1995). Sistemas sociales. Stanford: Prensa de la universidad de Stanford. Maturana, H. (1997). De Máquinas y Seres Vivos: autopoiesis de la organización de lo vivo. Santiago de Chile: Editorial Universitaria.

Ministerio de Medio Ambiente (2003). Sistema español de indicadores ambientales de turismo. Madrid: Ministerio de Medio Ambiente.

Ministério do Ambiente (1999). Turismo e ambiente: indicadores de integracao. Lisboa: Dirección General de Ambiente.

Morin, E. (1994). Introducción al pensamiento complejo. Barcelona: Gedisa.

Negrão, R. (2000). Desarrollo Sustentable. II Curso Internacional de Aspectos Geológicos de Protección Ambiental, UNESCO [En línea] [fecha de consulta 16 de mayo de 2015]. Disponible en http://www.ingenieroambiental.com/4014/desa-susten.pdf.

OECD (1993). OECD core set of indicators for environmental performance reviews. Environmental Monographs No. 83. Paris: Organization for Economic Cooperation and Development.

Ordaz, V. y Saldaña, G. (2005). Análisis y crítica de la Metodología para la realización de Planes Regionales en el estado de Guanajuato. Tesis (Maestría de Planeamiento Urbano Regional) Guanajuato, México: Universidad de Guanajuato. Facultad de Arquitectura. Departamento de postgrados. $346 \mathrm{p}$.

Organización Mundial de Turismo (2005). Previsiones del turismo mundial hasta el 
año 2006 y después. Madrid: OMT.

Organización Mundial de Turismo (1999). Guía para administraciones locales: desarrollo turístico sostenible. Madrid: OMT.

Panosso, A. (2007). "Filosofía del turismo: una propuesta epistemológica". Estudios y perspectivas del turismo, 16(4): 389-400.

Parsons de Talcott (1999). El sistema social. Madrid: Alianza.

Pujadas, R. y Font, J. (1998). Ordenación y Planeación Territorial. Madrid: Síntesis.

Rappoport, A. (1985). General Systems Theory. Cambridge: Abacus Press

Real Academia Española (2012). Diccionario de la lengua española (22 ed.). [En línea]. [Fecha de consulta 18 de mayo de 2015]. Disponible en http://buscon.rae.es/drae/?type= $3 \&$ val $=$ disquisici\%F3n\&val_aux $=$ \&origen $=$ REDRAE

Salinas, E. (2005). El Desarrollo Sustentable desde la Ecología del Paisaje. [En línea]. Cuba: CVGobernabilidad [fecha de consulta 18 de abril de 2015]. Disponible en http:// www.gobernabilidad.cl/modules.php?name $=$ News\&file $=$ print $\&$ sid $=796$

Secretaría de Turismo (2008). Agenda 21 para el Turismo Mexicano: un marco de acción para el desarrollo sustentable de la actividad turística. México: SECTUR.

Secretaría de Turismo (2007). Elementos para evaluar el impacto económico, social y ambiental de turismo de naturaleza en México. México: SECTUR.

Secretaría de Turismo (2004). Planeación y gestión del desarrollo turístico municipal: manual para la gestión del desarrollo turístico municipal. México: SECTUR.

Vera, J. (2001). Planificación y gestión del desarrollo turístico sostenible: propuestas para la creación de un sistema de indicadores. Documentos de trabajo. Instituto Universitario de Geografía, Universidad de Alicante. España.

Vignati, F. (2009). Gestión de destinos Turísticos: Cómo atraer a personas a polos, ciudades y países. México: Trillas. 